\title{
Modular Covariance of Minimal Model Correlation Functions
}

\author{
G. Felder ${ }^{1, \star}$ and R. Silvotti ${ }^{2, \star \star}$ \\ ${ }^{1}$ School of Mathematics, Institute for Advanced Study, Princeton, NJ 08540, USA \\ ${ }^{2}$ Theoretische Physik, Universität Zürich, Schönberggasse 9, CH-8001 Zürich, Switzerland
}

\begin{abstract}
We prove that one-point functions of all scaling fields in minimal left-right diagonal models of conformal field theory are modular covariant. This consistency condition should allow one to extend these minimal models to Riemann surfaces of arbitrary genus.
\end{abstract}

\section{Introduction}

In recent times, the problem of extending two-dimensional conformal field theory [1] to higher genus Riemann surfaces has received considerable attention [2,3]. The main reason for this interest lies in the fact that quantum corrections to classical string theories are given perturbatively in terms of correlation functions of some conformal field theory on Riemann surfaces of positive genus. On the other hand, the requirement of modular invariance of the partition function on the torus has turned out to be a simple but powerful tool to write down candidates for conformal invariant models $[4,5]$, giving a recipe for finding universality classes of statistical systems at the critical point.

In general, however, several important questions remain unanswered. Specifically, the partition function on the torus gives only the field content of a conformal invariant theory: Is there a consistent (crossing symmetric) operator product algebra corresponding to this field content? Can this theory consistently be extended to higher genus Riemann surfaces? Answering the first question requires solving the conformal bootstrap equations [1] and checking the consistency of the solution. These equations have been solved in some models [6,7]. As for the second question, there is general agreement $[8,9]$ that a necessary and sufficient condition for a consistent conformal field theory defined on the sphere to admit an extension to higher genus is that the one-point function of all scaling fields on the

\footnotetext{
* Supported in part by NSF grant DMS 8610730. Address after March 1989: Theoretische Physik, ETH-Hönggerberg, CH-8093 Zürich, Switzerland

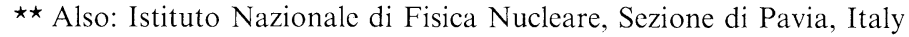


torus be modular covariant. Although the proof of the sufficiency of this condition may be cumbersome, depending on the way one constructs higher genus Green functions, the idea is quite intuitive: using crossing symmetry on the sphere, one may view a general punctured Riemann surface $X$ of genus $g$ as a punctured sphere with $g$ tori connected to it by tubes. Summing over all intermediate states propagating along the tubes gives Green functions on $X$ as infinite sum over products of Green functions on the sphere times one-point functions of secondary fields on the torus. Since the modular group is generated by Dehn twists along the cycles of the tori, it should be sufficient to check the covariance of one-point functions of all secondary fields under modular transformation.

In this paper, we study the modular transformation properties of the one-point conformal blocks for the discrete series of minimal models with central charge $c<1$ [1]. In a recent paper [10], an expression for the conformal blocks on the torus of minimal models in terms of free field vertex operators was given (see also [11-14] for related results). It is a generalization to genus one of the Feigin-Fuchs representation [6] of the conformal blocks. We show here that the one-point conformal blocks transform linearly into each other under modular transformations, and we compute the representation matrices of the generators $T$ and $S$. Inserting the Dotsenko-Fateev solution for the structure constants, we prove that the one-point function of primary fields of left-right symmetric models are modular covariant. We show, using the Eguchi-Ooguri Ward identities, that the same holds for all secondary fields. Thus left-right symmetric minimal models should be extendable to higher genus.

Our result for the representation matrices of modular transformations equally apply to the non-symmetric (A, D) and (A, E) models. The modular covariance of the corresponding one-point functions in these latter cases could be studied by inserting the structure constants computed in [15].

\section{Conformal Blocks on the Torus}

The primary chiral fields $\varphi_{N M}^{L}(z)$ of a minimal conformal field theory with central charge $c=1-6\left(p-p^{\prime}\right)^{2} / p p^{\prime}$, where $p$ and $p^{\prime}$ are positive relatively prime integers, are holomorphic operators mapping the irreducible Virasoro highest weight representation space (IHWS) $\mathscr{H}_{M}$ to the IHWS $\mathscr{H}_{L}$. An IHWS $\mathscr{H}_{N}$ is labeled by two indices $N=\left(n^{\prime}, n\right)$ belonging to the Kac table $F=\left\{1 \leqq n^{\prime} \leqq p^{\prime}-1\right.$, $1 \leqq n \leqq p-1\}$, and has highest conformal weight

$$
h_{n^{\prime} n}=h_{p^{\prime}-n^{\prime}, p-n}=\frac{\left(n^{\prime} p-n p^{\prime}\right)^{2}-\left(p-p^{\prime}\right)^{2}}{4 p p^{\prime}} \text {. }
$$

The operator $\varphi_{N M}^{L}(z)$ is uniquely determined up to a constant by its commutation relations with the Virasoro generators,

$$
\left[L_{k}, \varphi_{N M}^{L}(z)\right]=z^{k+1} \frac{d}{d z} \varphi_{N M}^{L}(z)+h_{N}(k+1) z^{k} \varphi_{N M}^{L}(z) .
$$


The primary fields of (left-right symmetric) minimal models are constructed as linear combinations of chiral fields in the $z$-variable times chiral fields in the $\bar{z}$ variable:

$$
\Phi_{N}(z, \bar{z})=\sum_{L M \in F_{0}} D_{N M}^{L} \varphi_{N M}^{L}(z) \otimes \varphi_{N M}^{L}(\bar{z})
$$

acting on $\bigoplus_{J \in F_{0}} \mathscr{H}_{J} \otimes \mathscr{H}_{J}$. Here $F_{0}$ is the fundamental domain $\left\{\left(n^{\prime}, n\right) \in F \mid p n^{\prime}<p^{\prime} n\right\}$. The coefficients $D_{N M}^{L}$ coincide with the structure constants of the operator product algebra if chiral primary fields are suitably normalized. We will however choose another normalization.

In [10] it was shown that the spaces $\mathscr{H}_{N}$ can be identified with the space of BRST states in a direct sum of Fock spaces of definite charge, for a suitable BRST operator $Q$, and that chiral primary field can be identified with BRST invariant "screened" vertex operators acting on these Fock spaces. Explicitly,

$$
\begin{aligned}
& \varphi_{N M}^{L}(z)=e_{N M}^{i \theta_{N M}^{L}} \int e^{i \alpha_{N} \varphi(z)} \prod_{j=1}^{r^{\prime}} e^{i \alpha_{-} \varphi\left(u_{j}^{\prime}\right)} d u_{j}^{\prime} \prod_{j=1}^{r} e^{i \alpha_{+} \varphi\left(u_{j}\right)} d u_{j}, \\
& \theta_{N M}^{L}=\frac{\pi}{2}\left\{\left(2 m^{\prime}+1\right) r+(2 m+1) r^{\prime}\right\}-\pi r^{\prime}\left(r^{\prime}-m^{\prime}\right) p / p^{\prime}-\pi r(r-m) p^{\prime} / p .
\end{aligned}
$$

The $U(1)$ charges are [6] $\alpha_{N}=\left(1-n^{\prime}\right) \alpha_{-} / 2+(1-n) \alpha_{+} / 2$, with screening charges $\alpha_{-}^{2}=p / p^{\prime}, \quad \alpha_{-} \alpha_{+}=-1$, and the free bosonic field is normalized as $\langle\varphi(z) \varphi(w)\rangle=-2 \log (z-w)$. The number of screening charges is determined by the neutrality condition $l=m+n-2 r-1, l^{\prime}=n^{\prime}+m^{\prime}-2 r^{\prime}-1$. The phase $\theta_{N M}^{L}$ was introduced to simplify further computations.

The Green functions of chiral primary fields (conformal blocks) on the torus were then computed in terms of traces of screened vertex operators over Fock spaces, with a suitable projection on BRST states. This construction gives an integral representation of conformal blocks on the torus. Here we are particularly interested in the one-point function. The torus is identified with the complex plane modulo the identification of $z$ with $q z, q=\exp 2 \pi i \tau,|q|<1$, and can be viewed as an annulus with identified boundaries. The unnormalized one-point function is then

$$
\operatorname{Tr} \Phi_{N}(z, \bar{z}) q^{L_{0}} \bar{q}^{\bar{L}_{0}}=\sum_{M} D_{N M}^{M} J_{M N}^{\mathrm{ann}}(z \mid \tau) J_{M N}^{\mathrm{ann}}(\bar{z} \mid-\bar{\tau}),
$$

with conformal blocks given by

$$
J_{M N}^{\mathrm{ann}}(z \mid \tau)=\operatorname{Tr}_{\mathscr{H}_{M}} \varphi_{N M}^{M}(z) q^{L_{0}} .
$$

In order to study modular properties of conformal blocks it is more convenient to represent the torus as $C / Z+\tau Z$ with complex coordinate $w$, related to $z$ by $z=\exp 2 \pi i w$. Taking into account the term coming from the Schwarzian derivative in the transformation of the energy momentum tensor, the conformal block in the variable $w$ is:

$$
J_{M N}(w \mid \tau)=\left(\frac{d z}{d w}\right)^{h_{N}} J_{M N}^{\mathrm{ann}}(z \mid \tau) q^{-c / 24} .
$$


This expression is independent of $w$, by translation invariance. At this point it is convenient to introduce the notation of [5]: to every pair $N=\left(n^{\prime}, n\right)$ in $F$ we assign an integer $v$ :

$$
v=n^{\prime} p-n p^{\prime} \text {. }
$$

Since $p$ and $p^{\prime}$ are relatively prime, $v$ determines the pair $n^{\prime}, n$ in $F$. There exists a number $\omega_{0}$ with $\omega_{0}^{2}=1 \bmod 4 p^{\prime} p$, depending on $p$ and $p^{\prime}$, such that $\omega_{0} v=n^{\prime} p+n p^{\prime} \bmod 2 p^{\prime} p$. In the following we will use both the notations $N, M$, $\ldots$ and $v, \mu, \ldots$, to label the representations. Upon using formula (3.40) of [10] after some calculations we get

$$
\begin{gathered}
J_{\mu, v}(\tau)=I_{\mu, v}(\tau)-I_{\omega_{0} \mu, v}(\tau), \\
I_{\mu, v}(\tau)=\frac{e^{i \theta_{, \mu}^{\mu}}}{\eta(\tau)} \int_{0}^{1} \prod_{i=1}^{r^{\prime}} d v_{i}^{\prime} \prod_{i=1}^{r} d v_{i} \Delta_{\mu, \varrho}(W \mid \tau) \Phi_{\varrho}\left(v_{1}^{\prime}, \ldots, v_{r^{\prime}}^{\prime}, v_{1}, \ldots, v_{r} \mid \tau\right), \\
\Phi_{\varrho}\left(v_{1}^{\prime}, \ldots, v_{r^{\prime}}^{\prime}, v_{1}, \ldots, v_{r} \mid \tau\right)=\prod_{1 \leqq i \leqq r^{\prime}} E\left(0, v_{i}^{\prime} \mid \tau\right)^{-2 \varrho / p^{\prime}} \prod_{1 \leqq i \leqq r} E\left(0, v_{i} \mid \tau\right)^{2 \varrho / p} \\
\cdot \prod_{1 \leqq i<j \leqq r^{\prime}} E\left(v_{i}^{\prime}, v_{j}^{\prime} \mid \tau\right)^{2 p / p^{\prime}} \prod_{1 \leqq i<j \leqq r} E\left(v_{i}, v_{j} \mid \tau\right)^{2 p^{\prime} / p} \\
\cdot \prod_{\substack{1 \leqq i^{\prime} \leqq r^{\prime} \\
1 \leqq i \leqq r}} E\left(v_{i^{\prime}}^{\prime}, v_{i} \mid \tau\right)^{-2},
\end{gathered}
$$

where

$$
\begin{aligned}
\Delta_{\mu \varrho}(W \mid \tau) & =\sum_{l \in \mathrm{Z}} e^{i \pi \tau\left(2 p^{\prime} p l+\mu\right)^{2} / 2 p^{\prime} p+i 4 \pi \varrho W\left(2 p^{\prime} p l+\mu\right) / 2 p^{\prime} p}, \\
W & =-\frac{1}{\varrho}\left(\sum_{i=1}^{r^{\prime}} p v_{i}^{\prime}-\sum_{i=1}^{r} p^{\prime} v_{i}\right),
\end{aligned}
$$

and the prime form on the torus is

$$
E(v, w \mid \tau)=\frac{\theta_{1}(v-w \mid \tau)}{\theta_{1}^{\prime}(0 \mid \tau)}
$$

The integration contours are all along the $a$-cycle. The numbers $r^{\prime}, r$ of screening charges are related to the representation labels by the formulae $r^{\prime}=\frac{1}{2}\left(n^{\prime}-1\right)$, $r=\frac{1}{2}(n-1)$. Note that if $n^{\prime}$ or $n$ is even, the one-point conformal block vanishes. The periodicity $\mu \rightarrow \mu+2 p^{\prime} p$ is apparent. Thus we can extend the range of $\mu$ in (2.9) to all integers modulo $2 p^{\prime} p$.

\section{The Representation of the Modular Group}

In this section we compute the behaviour of the one-point conformal blocks under modular transformations. The modular group is generated by two elements $T$ and $S$ acting on the upper half plane as $T: \tau \rightarrow \tau+1$ and $S: \tau \rightarrow-1 / \tau$. Under $T$ we have the transformation properties

$$
\begin{aligned}
\eta(\tau+1) & =e^{i \pi / 12} \eta(\tau), \\
\Delta_{\mu \varrho}(W \mid \tau+1) & =e^{i \pi \mu^{2} / 2 p^{\prime} p} \Delta_{\mu \varrho}(W \mid \tau), \\
E(v, w \mid \tau+1) & =E(v, w \mid \tau) .
\end{aligned}
$$


We conclude that

$$
J_{\mu \nu}(\tau+1)=e^{-i \pi / 12+i \pi \mu^{2} / 2 p^{\prime} p} J_{\mu \nu}(\tau) .
$$

The computation of the result of the $S$ transformation is done in two steps. In the first step $I_{\mu \nu}(-1 / \tau)$ is expressed as a linear combination of expressions with the same integrand as $I_{\lambda v}(\tau)$, but with screening charges integrated along the $b$-cycle rather than the $a$-cycle. In the second step the integration contours are deformed from the $b$ - to the $a$-cycle using the periodicity properties of the theta functions. The following formulae are well known:

$$
\begin{aligned}
\eta(-1 / \tau) & =(-i \tau)^{1 / 2} \eta(\tau), \\
E(v / \tau, w / \tau \mid-1 / \tau) & =\tau^{-1} e^{i \pi(v-w)^{2} / \tau} E(v, w \mid \tau) .
\end{aligned}
$$

The modular transformation properties of $\Delta_{\mu \varrho}$ are calculated in Appendix A. We have

$$
\Delta_{\mu \varrho}(W / \tau \mid-1 / \tau)=(-i \tau)^{1 / 2} e^{i 2 \pi \varrho^{2} W^{2} / \tau p^{\prime} p} \frac{1}{\sqrt{2 p^{\prime} p}} \sum_{\lambda=0}^{2 p^{\prime} p-1} e^{i \pi \mu \lambda / p^{\prime} p} \Delta_{-\lambda, \varrho}(W \mid \tau) .
$$

Inserting these formulae in (2.9), after the change of variables $v_{i}^{\prime} \rightarrow v_{i}^{\prime} / \tau, v_{i} \rightarrow v_{i} / \tau$, gives

$$
e^{-i \theta_{v \mu}^{\mu}} I_{\mu v}(-1 / \tau)=\frac{\tau^{h_{v}}}{\sqrt{2 p^{\prime} p}} \sum_{\lambda=0}^{2 p^{\prime} p-1} e^{i \pi \lambda \mu / p^{\prime} p} e^{-i \theta_{v}^{-2},} I_{-\lambda v}^{(b)}(\tau),
$$

where $I_{\mu \nu}^{(b)}(\tau)$ is given by the same expression (2.9) as $I_{\mu \nu}(\tau)=I_{\mu \nu}^{(a)}(\tau)$ but the integration contours are homologous to the $b$-cycle.

We come now to the second step of the computation of the $S$ transformation: we express the integrals $I^{(b)}$ in terms of the integrals $I^{(a)}$ by deforming one contour at a time. Since we are dealing with multivalued integrands we must be a little more precise in the definitions.

The integrand in the definition of $I_{\mu \nu}(\tau)$ is a multivalued function in $r^{\prime}+r$ integration variables. It is integrated over a simply connected manifold (a product of paths). Therefore $I_{\mu \nu}(\tau)$ is completely defined if we fix a point $P_{0}$ in $M=C^{r^{\prime}+r}-\bigcup_{i<j}\left\{z_{i}=z_{j}\right\}$, where we specify the value of the integrand, and give a homotopy class of paths $[\eta]$ in $M$ going from $P_{0}$ to a generic point $P$ of the manifold. In this way the integrand is defined as an analytic continuation from $P_{0}$ along any path in $[\eta]$. As $P_{0}$ we choose a point where all variables are ordered on the negative real axis $\left(v_{1}^{\prime}<\ldots<v_{r^{\prime}}^{\prime}<v_{1}<\ldots<v_{r}<0\right)$ so that at $P_{0}$ all arguments of the theta functions are positive. We fix the value of the integrand at $P_{0}$ by requiring that it be real at $\tau=i$.

Using this procedure we can define $I_{\mu \nu}^{(a)}(\tau), I_{\mu \nu}^{(b)}(\tau)$, and more generally $I_{\mu \nu}^{\left[s^{\prime}, s\right]}(\tau)$, defined as

$$
\begin{aligned}
I_{\mu \nu}^{\left[s^{\prime}, s\right]}(\tau)= & \frac{e^{i \theta_{v \mu}^{\mu}}}{\eta(\tau)} \int_{0}^{\tau} d v_{1}^{\prime} \ldots d v_{s^{\prime}}^{\prime} d v_{1} \ldots d v_{s} \int_{0}^{1} d v_{s^{\prime}+1}^{\prime} \ldots d v_{r^{\prime}}^{\prime} d v_{s+1} \ldots d v_{r} \\
& \cdot \Delta_{\mu \varrho}(W \mid \tau) \Phi_{\varrho}\left(v_{1}^{\prime}, \ldots, v_{r^{\prime}}^{\prime}, v_{1}, \ldots, v_{r} \mid \tau\right) .
\end{aligned}
$$




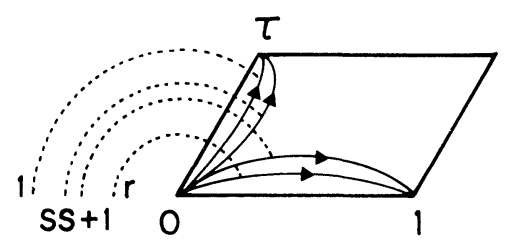

Fig. 1

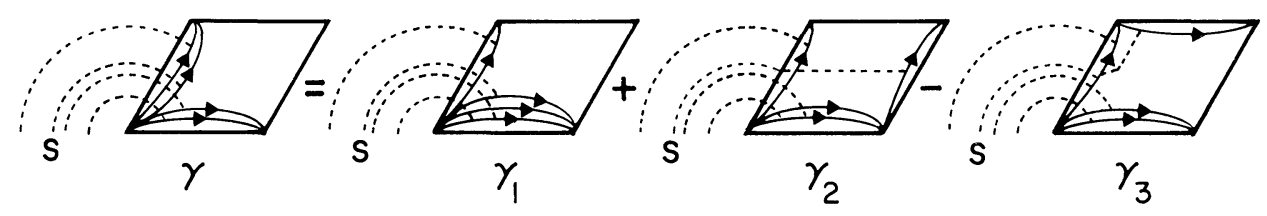

Fig. 2
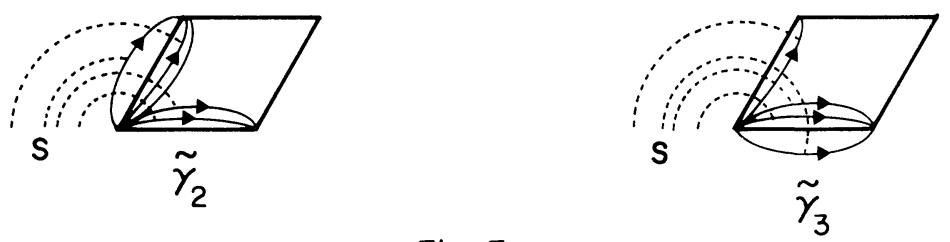

Fig. 3

The integration contours of the $v$ variables are drawn in Fig. 1. The dashed lines indicate the analytic continuation path $\eta$. A similar picture for the $v^{\prime}$ variables should be superimposed. The relative position of $v$ and $v^{\prime}$ integration contours does not play a role [6]. To derive a recursion relation, let us deform the contour of integration of the variable $v_{s}$ in $I_{\mu \nu}^{\left[s^{\prime}, s\right]}(\tau)$ into the sum of three contours as shown in Fig. 2. After a change of variables we get

$$
\begin{aligned}
I_{\mu \nu}^{\left[s^{\prime}, s\right]}(\tau)= & I_{\mu \nu}^{\left[s^{\prime}, s-1\right]}(\tau)+\frac{e^{i \theta_{v \mu}^{\mu}}}{\eta(\tau)} \int_{\bar{\gamma}_{2}} d v^{\prime} d v \Delta_{\mu \varrho}\left(W+p^{\prime} / \varrho \mid \tau\right) \Phi_{\varrho}\left(\ldots, v_{s}+1, \ldots \mid \tau\right) \\
& -\frac{e^{i \theta_{v \mu}^{\mu}}}{\eta(\tau)} \int_{\tilde{\gamma}_{3}} d v^{\prime} d v \Delta_{\mu \varrho}\left(W+p^{\prime} \tau / \varrho \mid \tau\right) \Phi_{\varrho}\left(\ldots, v_{s}+\tau, \ldots \mid \tau\right)
\end{aligned}
$$

The integration contours $\tilde{\gamma}_{2}$ and $\tilde{\gamma}_{3}$ are depicted in Fig. 3. The value of $\Phi_{\varrho}(\ldots$, $\left.v_{s}+1, \ldots \mid \tau\right)$ at $P$ is the analytic continuation of $\Phi_{\varrho}\left(\ldots, v_{s}, \ldots \mid \tau\right)$ along the path $\tilde{\eta}_{2}^{-1} \circ \eta_{2}, \eta_{2}$ and $\tilde{\eta}_{2}$ being the analytic continuation paths in Figs. 2, 3. This path $\tilde{\eta}_{2}^{-1} \circ \eta_{2}$ leaves all variables fixed except $v_{s}$ that moves along a straight line joining $v_{s}$ to $v_{s}+1$. The behaviour of the theta function to fractional powers under this analytic continuation is computed in Appendix B. We obtain

$$
\Phi_{\varrho}\left(v_{1}^{\prime}, \ldots, v_{s}+1, \ldots, v_{r} \mid \tau\right)=e^{i \pi(s-1) 2 p^{\prime} / p} e^{-i \pi(r-s) 2 p^{\prime} / p} e^{-i \pi 2 \varrho / p} \Phi_{\varrho}\left(v_{1}^{\prime}, \ldots, v_{r} \mid \tau\right) .
$$


The same applies to the second integrand in (3.7), with the result

$$
\Phi_{\varrho}\left(v_{1}^{\prime}, \ldots, v_{s}+\tau, \ldots, v_{r} \mid \tau\right)=e^{i \pi(r-1) 2 p^{\prime} / p} e^{i \pi 2 \varrho / p} e^{2 i \pi \tau p^{\prime} / p+4 i \pi \varrho W / p} \Phi_{\varrho}\left(v_{1}^{\prime}, \ldots, v_{r} \mid \tau\right) \text {. }
$$

The properties of $\Delta_{\mu \varrho}(W \mid \tau)$ under translations of the argument are computed in Appendix A. We have

$$
\begin{aligned}
\Delta_{\mu \varrho}\left(W+p^{\prime}|\varrho| \tau\right) & =e^{2 \pi i \mu / p} \Delta_{\mu \varrho}(W \mid \tau), \\
\Delta_{\mu \varrho}\left(W+p^{\prime} \tau / \varrho \mid \tau\right) & =e^{-i 2 \pi \tau p^{\prime} / p-i 4 \pi \varrho W / p} \Delta_{\mu+2 p^{\prime}, \varrho}(W \mid \tau) .
\end{aligned}
$$

To bring the analytic continuation paths $\tilde{\eta}_{2}, \tilde{\eta}_{3}$ to the standard form of Fig. 1 we get a phase $\exp \left(-i \pi(s-1) 2 p^{\prime} / p\right), \exp \left(-i \pi(r-s) 2 p^{\prime} / p\right)$, respectively. The end result can be put in a nicer form by introducing the quantity $i_{\mu \nu}^{\left[s^{\prime}, s\right]}(\tau)$ defined by

$$
I_{\mu v}^{\left[s^{\prime}, s\right]}(\tau)=e^{-i \pi \sigma(\sigma-2 \mu+\varepsilon) / 2 p^{\prime} p} i_{\mu-\sigma, v}^{\left[s^{\prime}, s\right]}(\tau),
$$

with $\sigma=s^{\prime} p-s p^{\prime}, \varepsilon=p-p^{\prime}$. In this notation we obtain, after collecting all the factors,

$$
2 \sin (\pi \mu / p) i_{\mu \nu}^{\left[s^{\prime}, s\right]}(\tau)=i_{\mu-p^{\prime}, v}^{\left[s^{\prime}, s-1\right]}(\tau)-i_{\mu+p^{\prime}, v}^{\left[s^{\prime}, s-1\right]}(\tau) .
$$

A completely analogous calculation can be carried out for the deformation of a $v^{\prime}$ integration contour. The resulting recursion is

$$
2 \sin \left(\pi \mu / p^{\prime}\right) i_{\mu \nu}^{\left[s^{\prime}, s\right]}(\tau)=i_{\mu-p, v}^{\left[s^{\prime}-1, s\right]}(\tau)-i_{\mu+p, v}^{\left[s^{\prime}-1, s\right]}(\tau) .
$$

We recall that the one-point conformal block is equal to $i_{\mu, v}^{[0,0]}(\tau)-i_{\omega_{0} \mu, v}^{[0,0]}(\tau)$. The following symmetry properties will be important later:

$$
i_{-\mu \nu}^{[0,0]}(\tau)=i_{\mu \nu}^{[0,0]}(\tau), \quad i_{-\mu \nu}^{\left[r^{\prime}, r\right]}(\tau)=i_{\mu \nu}^{\left[r^{\prime}, r\right]}(\tau) .
$$

They are obtained by the change of variables $v_{i}^{\prime} \rightarrow 1-v_{r^{\prime}-i+1}^{\prime}, v_{i} \rightarrow 1-v_{r-i+1}$ and $v_{i}^{\prime} \rightarrow \tau-v_{r^{\prime}-i+1}^{\prime}, v_{i} \rightarrow \tau-v_{r-i+1}$, respectively.

It is a remarkable fact that as a by-product of this construction we can rederive the fusion rules $[1,10]$, as consequence of the following identities:

$$
\begin{aligned}
& \text { If } 1 \leqq m \leqq r-s \quad \text { then } \quad i_{\mu \nu}^{\left[s^{\prime}, s\right]}(\tau)=i_{\omega_{0} \mu, v}^{\left[s^{\prime}, s\right]}(\tau) \text {, } \\
& \text { If } 1 \leqq m^{\prime} \leqq r^{\prime}-s^{\prime} \quad \text { then } \quad i_{\mu \nu}^{\left[s^{\prime}, s\right]}(\tau)=i_{-\omega_{0} \mu, \nu}^{\left[s^{\prime}, s\right]}(\tau) \text {. }
\end{aligned}
$$

The fusion rules arise in the special case $\left[s^{\prime}, s\right]=[0,0]$. In this case we see, using (3.14) that the one-point conformal block $J_{\mu \nu}^{(a)}$ vanishes if $1 \leqq m \leqq r$, or $1 \leqq m^{\prime} \leqq r^{\prime}$. Using (3.14) again and the fact that $\left(m^{\prime}-p^{\prime}, m-p\right)$ and $\left(m^{\prime}, m\right)$ correspond to the same value of $\mu$, we see that $J_{\mu \nu}^{(a)}$ also vanishes if $1 \leqq p-m \leqq r$ or $1 \leqq p^{\prime}-m^{\prime} \leqq r^{\prime}$. We recover the fusion rule, that in this case states that $J_{\mu \nu}^{(a)}$ can be non-vanishing only if

$$
\begin{gathered}
\frac{n+1}{2} \leqq m \leqq p-\frac{n+1}{2}, \\
\frac{n^{\prime}+1}{2} \leqq m^{\prime} \leqq p^{\prime}-\frac{n^{\prime}+1}{2} .
\end{gathered}
$$

The proof of $(3.15 \mathrm{a}, \mathrm{b})$ is by induction on $m^{\prime}, m$. To prove the claim for $m=1$, set $\mu=m^{\prime} p$ in (3.14). The left-hand side vanishes and (3.15a) results. We assume now 
that the claim is proven up to a specific $m$ and want to prove it for $m+1$. Multiplying the induction hypothesis (3.15a) by $\sin (\pi \mu / p)$, and using the recursion relation on both sides gives

$$
i_{m^{\prime} p-(m+1) p^{\prime}, v}^{\left[s^{\prime}, s-1\right]}-i_{m^{\prime} p-(m-1) p^{\prime}, v}^{\left[s^{\prime}, s-1\right]}=-\left(i_{m^{\prime} p+(m-1) p^{\prime}, v}^{[s, s-1]}-i_{m^{\prime} p+(m+1) p^{\prime}, v}^{\left[s^{\prime}, s-1\right]}\right) .
$$

The claim follows by using the induction hypothesis for $m-1$. The identity $(3.15 \mathrm{~b})$ is proven exactly the same way.

We are now ready to give the representation matrix of the $S$ transformation. The quantity

$$
j_{\mu \nu}^{\left[s^{\prime}, s\right]}(\tau)=i_{\mu \nu}^{\left[s^{\prime}, s\right]}(\tau)-i_{\omega_{0} \mu, \nu}^{\left[s^{\prime}, s\right]}(\tau)
$$

coincides with the one-point conformal block if $s=s^{\prime}=0$. Equation (3.5) can be rewritten as

$$
j_{\mu \nu}^{[0,0]}(-1 / \tau)=(-i \tau)^{h_{v}} \frac{1}{\sqrt{2 p^{\prime} p}} \sum_{\lambda=0}^{2 p^{\prime} p-1} e^{-i \pi \lambda \mu / p^{\prime} p} j_{\lambda \nu}^{\left[r^{\prime}, r\right]}(\tau) .
$$

Using the symmetries (3.14) we can reduce the sum to the fundamental domain:

$$
\begin{aligned}
j_{\mu \nu}^{[0,0]}(-1 / \tau)= & (-i \tau)^{h_{v}} \sqrt{\frac{2}{p^{\prime} p}} \sum_{\left(l^{\prime} l\right) \in F_{0}}(-1)^{l^{\prime} m+l m^{\prime}+1} \sin \left(\pi l^{\prime} m^{\prime} p / p^{\prime}\right) \\
& \cdot \sin \left(\pi l m p^{\prime} / p\right) j_{\lambda \nu}^{\left[r^{\prime}, r\right]}(\tau) .
\end{aligned}
$$

The representation matrix of the $S$ transformation is defined as

$$
J_{\mu \nu}^{(a)}(-1 / \tau)=(-i \tau)^{h_{\nu}} \sum_{\lambda=0}^{2 p^{\prime} p-1} S_{\mu \lambda} J_{\lambda \nu}^{(a)}(\tau)
$$

The summation is over all $\lambda$ for which the fusion rules are obeyed. From (3.19) and the recursion relations $(3.12),(3.13)$, we get

$$
S=U A^{\prime r^{\prime}} A^{r}
$$

where $U$ is the Fourier transformation matrix

$$
U_{\mu \lambda}=\frac{1}{\sqrt{2 p^{\prime} p}} e^{-i \pi \mu \lambda / p^{\prime} p}
$$

and $A^{\prime}, A$ are commuting matrices given by

$$
\begin{aligned}
& A_{\mu \lambda}=(2 \sin \pi \mu / p)^{-1}\left(\delta_{\mu, \lambda+p^{\prime}}-\delta_{\mu, \lambda-p^{\prime}}\right), \\
& A_{\mu \lambda}^{\prime}=\left(2 \sin \pi \mu / p^{\prime}\right)^{-1}\left(\delta_{\mu, \lambda+p}-\delta_{\mu, \lambda-p}\right) .
\end{aligned}
$$

A more explicit expression can be given for the product $A^{\prime r^{\prime}} A^{r}$. Let us introduce the notation

$$
\begin{aligned}
{[j] } & =2 \sin \left(\pi j p^{\prime} / p\right) \\
{\left[\begin{array}{c}
m \\
n
\end{array}\right] } & =\frac{[m][m-1] \cdots[m-n+1]}{[n][n-1] \cdots[1]} .
\end{aligned}
$$

Then

$$
\left(A^{\prime r^{\prime}} A^{r}\right)_{\mu \lambda}=(-1)^{m^{\prime} r+m r^{\prime}}\left(a^{\prime r^{\prime}}\right)_{m^{\prime} l^{\prime}}\left(a^{r}\right)_{m l}
$$


where

$$
\left(a^{r}\right)_{m l}=(-1)^{\frac{1}{2}(m-r-l)}\left[\begin{array}{c}
r \\
\frac{1}{2}(m+r-l)
\end{array} \prod_{\substack{j=\frac{1}{2}(m+l-r) \\
j \neq l}}^{\frac{1}{2}(m+l+r)}[j]^{-1},\right.
$$

if $|m-l| \leqq r, m+l+r=0 \bmod 2$, and vanishes otherwise. The matrix $a^{\prime r^{\prime}}$ is given by the same expression but with $[j]$ replaced by $[j]^{\prime}=2 \sin \left(\pi j p / p^{\prime}\right)$. These formulae can be easily proven by induction on $r$ and $r^{\prime}$. Note that it is sufficient to compute the matrix elements $S_{\mu \lambda}$ for $\mu, \lambda$ obeying the fusion rules. For these values there are never divergent denominators in (3.27).

\section{Modular Covariance of One-Point Functions of Primary Fields}

Consider the one-point function of the primary field $\Phi_{N}, N=\left(n^{\prime}, n\right)$, of the leftright symmetric models. On the torus $C / Z+\tau Z$ :

$$
\left\langle\Phi_{N}(0)\right\rangle(\tau)=\frac{1}{Z(\tau)} \sum_{M} D_{N M}^{M} J_{M N}^{(a)}(\tau) J_{M N}^{(a)}(-\bar{\tau}) .
$$

The partition function $Z(\tau)$ is modular invariant. In our normalization, the $D$ coefficients read $[6,16]$

$$
D_{N M}^{M}=\Delta_{N} \prod_{\substack{j^{\prime}=m^{\prime}-r^{\prime} \\ j^{\prime} \neq m^{\prime}}}^{m^{\prime}+r^{\prime}}\left[j^{\prime}\right]^{\prime-1} \prod_{\substack{j=m-r \\ j \neq m}}^{m+r}[j]^{-1}
$$

with an $M$ independent factor $\Delta_{N}$. As before, $r^{(\prime)}=\left(n^{(\prime)}-1\right) / 2$. The property of modular covariance of the one-point function

$$
\left\langle\Phi_{N}(0)\right\rangle(-1 / \tau)=|\tau|^{2 h_{N}}\left\langle\Phi_{N}(0)\right\rangle(\tau),
$$

is equivalent to the condition $S^{T} D S=D$. In the notation of the last section, $D$ is the diagonal matrix

$$
D_{\mu \lambda}=\delta_{\mu \lambda} D_{N M}^{M}
$$

and we think of $N$ as fixed in these considerations. This modular covariance equation could be used to determine the $D$ coefficients in (4.1). Here we verify that the Dotsenko-Fateev solution, obtained by imposing crossing symmetry on the plane, gives the right result. Using the fact that $S^{2}=1$ once projected to the fundamental domain, we can rewrite this equation in the more convenient form

$$
\left(S D^{-1}\right)^{T}=S D^{-1} \text {. }
$$

The matrix $S D^{-1}$ can be computed using the explicit formulae derived in Sect. 3 . In the variables $x=\exp \left(2 \pi i p^{\prime} / p\right), x^{\prime}=\exp \left(2 \pi i p / p^{\prime}\right)$, we have

$$
\begin{aligned}
\left(S D^{-1}\right)_{\mu \lambda} & =\Delta_{N}^{-1} \sqrt{\frac{2}{p^{\prime} p}} t_{m^{\prime} l^{\prime}}^{\prime} t_{m l}(-1)^{\left(m^{\prime}+r^{\prime}\right)(l+r)+(m+r)\left(l^{\prime}+r^{\prime}\right)}, \\
t_{m l} & =(-1)^{r} x^{m l / 2} \prod_{j=1}^{r}[j] \sum_{j=0}^{r} x^{m(j-r / 2)}\left[\begin{array}{c}
r+l \\
r-j
\end{array}\right]\left[\begin{array}{c}
r-l \\
j
\end{array}\right],
\end{aligned}
$$


and the same expression with primes holds for $t_{m^{\prime} n^{\prime}}^{\prime}$. Thus we have reduced our problem to the identities $t_{m l}=t_{l m}, t_{m^{\prime} l^{\prime}}^{\prime}=t_{l^{\prime} m^{\prime}}^{\prime}$. To prove this identity we notice that the Laurent polynomial in $x$

$$
\sum_{j=0}^{r} x^{m(j-r / 2)}\left[\begin{array}{c}
r+l \\
r-j
\end{array}\right]\left[\begin{array}{c}
r-l \\
j
\end{array}\right],
$$

is the coefficient of $t^{r}$ of the series

$$
\sum_{j_{1}, j_{2}=0}^{\infty} x^{m\left(j_{2}-j_{1}\right) / 2} t^{j_{1}+j_{2}}\left[\begin{array}{c}
r+\eta \\
j_{1}
\end{array}\right]\left[\begin{array}{c}
r-\eta \\
j_{2}
\end{array}\right] .
$$

Using the "q-generalization of the binomial formula" [17]

$$
\sum_{j=0}^{\infty}\left[\begin{array}{l}
n \\
j
\end{array}\right] t^{j}=\prod_{j=0}^{\infty} \frac{1+t x^{j+(1-n) / 2}}{1+t x^{j+(1+n) / 2}},
$$

this series can be brought to the form

$$
(4.8)=\prod_{j=0}^{\infty} \frac{\left(1+t x^{j+(l-r+m+1) / 2}\right)\left(1+t x^{j+(-l-r-m+1) / 2}\right)}{\left(1+t x^{j+(l+r-m+1) / 2}\right)\left(1+t x^{j+(-l+r+m+1) / 2}\right)},
$$

which is manifestly symmetric under the interchange of $m$ and $l$. Since this identity of formal power series in $t$ is valid for all $x$ (the product has effectively finitely many factors), both needed identities are proven. The proof of modular covariance is complete.

\section{Modular Covariance of One-Point Functions of Secondary Fields}

The unnormalized one-point function of a secondary field is

$$
\operatorname{Tr}\left(\Phi_{N}^{\{k, \bar{k}\}}(z, \bar{z}) q^{L_{0}} \bar{q}^{\bar{L}_{0}}\right)=\sum_{M} D_{N M}^{M} J_{M N}^{\{k\} \text { ann }}(z \mid \tau) J_{M N}^{\{\bar{k}\} \text { ann }}(\bar{z} \mid-\bar{\tau})
$$

with conformal blocks

$$
J_{M N}^{\{k\} \text { ann }}(z \mid \tau)=\operatorname{Tr}_{\mathscr{H}_{M}}\left[\left(L_{-k_{1}} \cdots L_{-k_{s}} \varphi_{N M}^{M}\right)(z) q^{L_{0}}\right]
$$

By Ward identities, the right-hand side can be represented in terms of a differential operator acting on the conformal block (2.6) of the primary field $\Phi_{N}(z, \bar{z})$. In the coordinate $w$ on $C / Z+\tau Z$ :

$$
J_{M N}^{\{k\}}(w \mid \tau)=D_{\tau}^{\{k\}} J_{M N}(w \mid \tau) .
$$

Due to translation invariance, the above is independent of $w$ and $D_{\tau}^{\{k\}}$ is a differential operator in $\tau$ only. In this section, we show that

$$
J_{M N}^{\{k\}}(\tau+1)=e^{-i \pi / 12+i \pi \mu^{2} / 2 p^{\prime} p} J_{M N}^{\{k\}}(\tau),
$$




$$
J_{M N}^{\{k\}}(-1 / \tau)=(-i \tau)^{h_{N}} \tau^{K} \sum_{L} S_{M L} J_{L N}^{\{k\}}(\tau)
$$

with the matrix $S$ as in (3.22) and $K=\sum_{i=1}^{s} k_{i}$. The modular covariance of (5.1) then follows from the results of Sect. 4. In particular, the one-point function is invariant under $T$ and

$$
\left\langle\Phi_{N}^{\{k, \bar{k}\}}(w / \tau, \bar{w} / \bar{\tau})\right\rangle(-1 / \tau)=\tau^{h_{N}+K} \bar{\tau}^{h_{N}+\bar{K}}\left\langle\Phi_{N}^{\{k, \bar{k}\}}(w, \bar{w})\right\rangle(\tau) .
$$

Identity (5.4) is an immediate consequence of the fact that the eigenvalues of $L_{0}$ on $\mathscr{H}_{M}$ are equal to $h_{M}$ modulo one, and (5.5) follows from (3.21) and from the transformation properties of the differential operator $D_{\tau}^{\{k\}}$. To compute the latter we generalize the Eguchi-Ooguri [3] Ward identities on the torus to an arbitrary number of energy momentum tensor insertions. We prove these identities in the operator formalism, using the KMS property, as in [18].

Let us consider a product of (not necessarily primary) fields $F\left(z_{1}, \ldots\right.$, $\left.z_{n}\right)=\psi_{1}\left(z_{1}\right) \cdots \psi_{n}\left(z_{n}\right)$. By using the cyclic property of the trace and the commutation rule $L_{k} q^{L_{0}}=q^{L_{0}+k} L_{k}$, we obtain

$$
\operatorname{Tr} L_{k} F\left(z_{1}, \ldots, z_{n}\right) q^{L_{0}}=\frac{1}{1-q^{k}} \operatorname{Tr}\left[L_{k}, F\left(z_{1}, \ldots, z_{n}\right)\right] q^{L_{0}},
$$

for $k \neq 0$. For $k=0$,

$$
\operatorname{Tr} L_{0} F\left(z_{1}, \ldots, z_{n}\right) q^{L_{0}}=q \frac{\partial}{\partial q} \operatorname{Tr} F\left(z_{1}, \ldots, z_{n}\right) q^{L_{0}}
$$

To find the expectation value of $T(u) F\left(z_{1}, \ldots, z_{n}\right)=\sum_{k \in Z} u^{-k-2} L_{k} F\left(z_{1}, \ldots, z_{n}\right)$, we first represent the commutator on the right-hand-side of (5.7) in the usual form $(2 \pi i)^{-1} \oint_{\gamma} d z z^{k+1} T(z) F\left(z_{1}, \ldots, z_{n}\right)$, with the contour $\gamma$ encircling the points $z_{1}, \ldots, z_{n}$ and homologous to the trivial cycle of the torus. Then we note that

$$
\sum_{z \neq 0} \frac{1}{1-q^{k}}\left(\frac{z}{u}\right)^{k}=-\frac{1}{2 \pi i} \zeta(w-v)+\frac{1}{\pi i} \eta_{1} \cdot(w-v)-\frac{1}{2},
$$

where $z=e^{2 \pi i w}, u=e^{2 \pi i v} ; \zeta(w)=\theta_{1}^{\prime}(w \mid \tau) / \theta_{1}(w \mid \tau)+2 \eta_{1} \cdot w$ is the Weierstrass zeta function, and $\eta_{1}=\zeta(1 / 2)$. Finally, we transform to the coordinates on $C / Z+\tau Z$, with the result

$$
\begin{aligned}
& \operatorname{Tr} T(v) F\left(w_{1}, \ldots, w_{n}\right) q^{L_{0}-c / 24}=2 \pi i \frac{\partial}{\partial \tau} \operatorname{Tr} F\left(w_{1}, \ldots, w_{n}\right) q^{L_{0}-c / 24} \\
& +\frac{1}{2 \pi i} \oint_{\gamma} d w\left(-\zeta(w-v)+2 \eta_{1}\right. \\
& \quad(w-v)-\pi i) \operatorname{Tr} T(w) F\left(w_{1}, \ldots, w_{n}\right) q^{L_{0}-c / 24}
\end{aligned}
$$


Consider the case where the first $s$ fields in $F$ are energy momentum tensors and the remaining $m=n-s$ are chiral primary fields. Using the operator product expansion to perform the contour integral in (5.10), we obtain the recursion

$\operatorname{Tr} T(v) T\left(v_{1}\right) \cdots T\left(v_{s}\right) \varphi_{1}\left(w_{1}\right) \cdots \varphi_{m}\left(w_{m}\right) q^{L_{0}-c / 24}$

$$
\begin{aligned}
= & \left\{2 \pi i \frac{\partial}{\partial \tau}+\sum_{j=1}^{s}\left[2\left(\mathscr{P}\left(v-v_{j}\right)+2 \eta_{1}\right)+\left(\zeta\left(v-v_{j}\right)+2 \eta_{1} v_{j}\right) \frac{\partial}{\partial v_{j}}\right]\right. \\
& \left.+\sum_{i=1}^{m}\left[h_{i}\left(\mathscr{P}\left(v-w_{i}\right)+2 \eta_{1}\right)+\left(\zeta\left(v-w_{i}\right)+2 \eta_{1} w_{i}\right) \frac{\partial}{\partial w_{i}}\right]\right\} \\
& \cdot \operatorname{Tr} T\left(v_{1}\right) \cdots T\left(v_{s}\right) \varphi_{1}\left(w_{1}\right) \cdots \varphi_{m}\left(w_{m}\right) q^{L_{0}-c / 24} \\
& +\sum_{j=1}^{s} \frac{c}{12} \mathscr{P}^{\prime \prime}\left(v-v_{j}\right) \operatorname{Tr} T\left(v_{1}\right) \cdots \hat{T}\left(v_{j}\right) \cdots T\left(v_{s}\right) \varphi_{1}\left(w_{1}\right) \cdots \varphi_{m}\left(w_{m}\right) q^{L_{0}-c / 24},
\end{aligned}
$$

where $\mathscr{P}(w)=-\zeta^{\prime}(w)$ is the Weierstrass $\mathscr{P}$ function, and ${ }^{\wedge}$ denotes omission. In the case $s=1,2$, we recover the Eguchi-Ooguri identities on the torus.

Using the well known transformation properties $\zeta(v / \tau \mid-1 / \tau)=\tau \zeta(v \mid \tau)$, $\eta_{1}(-1 / \tau)=\tau^{2} \eta_{1}(\tau)-\pi i \tau$, we can deduce recursively from this Ward identity the modular transformation properties of one-point conformal blocks with $s$ insertions of the energy momentum tensor:

$$
\begin{aligned}
& \operatorname{Tr}_{\mathscr{H}_{M}} T\left(\frac{v_{1}}{\tau}\right) \cdots T\left(\frac{v_{s}}{\tau}\right) \varphi_{N M}^{M}\left(\frac{w}{\tau}\right) e^{2 \pi i(-1 / \tau)\left(L_{0}-c / 24\right)} \\
& =(-i \tau)^{h_{n}} \tau^{2 s} \sum_{L} S_{M L} \operatorname{Tr}_{\mathscr{H}_{L}} T\left(v_{1}\right) \cdots T\left(v_{s}\right) \varphi_{N L}^{L}(w) e^{2 \pi i \tau\left(L_{0}-c / 24\right)}
\end{aligned}
$$

This identity is equivalent to (5.5) as can be seen by representing the secondary fields as multiple integrals of expressions (5.11) [1].

\section{Appendix A. Properties of $\Delta_{\mu \rho}(W \mid \tau)$}

The function

$$
\Delta_{\mu \varrho}(W \mid \tau)=\sum_{l \in Z} e^{i \pi \tau\left(2 p^{\prime} p l+\mu\right)^{2} / 2 p^{\prime} p+i 4 \pi \varrho W\left(2 p^{\prime} p l+\mu\right) / 2 p^{\prime} p}, \quad \mu, \varrho \in Z
$$

is a generalization of Jacobi's theta function studied by Hermite (see [19]). It is periodic in $\mu: \Delta_{\mu \varrho}(W \mid \tau)=\Delta_{\mu+2 p^{\prime} p, \varrho}(W \mid \tau)$ and $\Delta_{\mu \varrho}(W \mid \tau)=\Delta_{-\mu \varrho}(-W \mid \tau)$.

For $W$ as in (2.10), translations of the arguments $v_{i}$ (or $v_{i}^{\prime}$ ) along a cycle $a$ or $b$ correspond to translations of $W$ by multiples of $p^{\prime} / \varrho, p^{\prime} \tau / \varrho($ or $p / \varrho, p \tau / \varrho)$. A simple calculation yields

$$
\begin{aligned}
\Delta_{\mu \varrho}\left(W+\frac{\lambda}{2 \varrho} \mid \tau\right) & =e^{i \pi \lambda \mu / p^{\prime} p} \Delta_{\mu \varrho}(W \mid \tau), \\
\Delta_{\mu \varrho}\left(W+\frac{\lambda}{2 \varrho} \tau \mid \tau\right) & =e^{-i \pi \lambda^{2} \tau / 2 p^{\prime} p-i 2 \pi \lambda \varrho W / p^{\prime} p} \Delta_{\mu+\lambda, \varrho}(W \mid \tau),
\end{aligned}
$$

with $\lambda$ integer. For $\lambda=2 p^{\prime}$ we get (3.10). 
Under the modular transformation $T$,

$$
\Delta_{\mu \varrho}(W \mid \tau+1)=e^{i \pi \mu^{2} / 2 p^{\prime} p} \Delta_{\mu \varrho}(W \mid \tau) .
$$

To prove the transformation property under $S$, we consider the Fourier transform of $\Delta_{\mu \varrho}(W / \tau \mid-1 / \tau)$ :

$$
\begin{aligned}
\tilde{\Delta}_{\lambda \varrho}(W / \tau \mid-1 / \tau) & =\frac{1}{\sqrt{2 p^{\prime} p}} \sum_{\mu=0}^{2 p^{\prime} p-1} e^{-i \pi \lambda \mu / p^{\prime} p} \Delta_{\mu \varrho}(W / \tau \mid-1 / \tau) \\
& =\frac{1}{\sqrt{2 p^{\prime} p}} \sum_{\mu=0}^{2 p^{\prime} p-1} \Delta_{\mu \varrho}(W / \tau-\lambda / 2 \varrho \mid-1 / \tau) \\
& =\frac{1}{\sqrt{2 p^{\prime} p}} \sum_{n \in Z} e^{-i \pi n^{2} / 2 p^{\prime} p \tau+i 4 \pi \varrho(W-\lambda \tau / 2 \varrho) n / 2 p^{\prime} p \tau},
\end{aligned}
$$

where in the second step we use (A.2), and in the third the explicit form of $\Delta_{\mu \varrho}(W / \tau-\lambda / 2 \varrho \mid-1 / \tau)$, for $n=2 p^{\prime} p l+\mu$ ranges over all integers as $l$ ranges over $\mathrm{Z}$ and $\mu$ over one period. The last expression can be computed by Poisson's summation formula, with the result

$$
\begin{aligned}
\tilde{\Delta}_{\lambda \varrho}(W / \tau \mid-1 / \tau) & =(-i \tau)^{1 / 2} e^{i 2 \pi \varrho^{2}(W-\lambda \tau / 2 \varrho)^{2} / p^{\prime} p \tau} \sum_{l \in Z} e^{i 2 \pi p^{\prime} p \tau l^{2}+i 4 \pi \varrho(-W+\lambda \tau / 2 \varrho) l} \\
& =(-i \tau)^{1 / 2} e^{i 2 \pi \varrho^{2} W^{2} / p^{\prime} p \tau} \Delta_{-\lambda, \varrho}(W \mid \tau) .
\end{aligned}
$$

Finally, inverting the Fourier transform, we get

$$
\Delta_{\mu \varrho}(W / \tau \mid-1 / \tau)=(-i \tau)^{1 / 2} e^{i 2 \pi \varrho^{2} W^{2} / p^{\prime} p \tau} \frac{1}{\sqrt{2 p^{\prime} p}} \sum_{\lambda=0}^{2 p^{\prime} p-1} e^{i \pi \mu \hat{\lambda} / p^{\prime} p} \Delta_{-\lambda, \varrho}(W \mid \tau) .
$$

\section{Appendix B. Analytic Continuation of Fractional Powers of $\theta_{1}(w \mid \tau)$}

Consider the torus $C / Z+\tau Z$, described by the coordinate $w=x+\tau y$ ( $x$ and $y$ real), and the many-valued function $\theta_{1}(w \mid \tau)^{\alpha}$. We want to determine the phases $\varphi_{a}$ and $\varphi_{b}$ in the equations

$$
\begin{aligned}
& \theta_{1}(w+1 \mid \tau)^{\alpha}=e^{i \alpha \varphi_{a}} \theta_{1}(w \mid \tau)^{\alpha}, \\
& \theta_{1}(w+\tau \mid \tau)^{\alpha}=e^{i \alpha \varphi_{b}} e^{-i \pi \alpha \tau-2 i \pi \alpha w} \theta_{1}(w \mid \tau)^{\alpha} .
\end{aligned}
$$

We define $\theta_{1}(w+1 \mid \tau)^{\alpha}$ (or $\left.\theta_{1}(w+\tau \mid \tau)^{\alpha}\right)$ as the analytic continuation of $\theta_{1}(w \mid \tau)^{\alpha}$ along a straight path going from $w$ to $w+1$ (or $w+\tau$ ). Note that $\theta_{1}(w \mid \tau)$ vanishes only at the lattice points $w_{m n}=m+n \tau, m, n \in \mathrm{Z}$, and we have

$$
\oint_{i m+n t} \theta_{1}^{\prime}(w \mid \tau) / \theta_{1}(w \mid \tau) d w=2 \pi i
$$

as $\gamma_{m+n \tau}$ encircles one lattice point with positive orientation.

To compute the phase in the first of Eqs. (B.1), we assume $0<y<1$ and consider the integral

$$
i \varphi_{a}=\int_{w}^{w+1} d \log \theta_{1}(v \mid \tau)=\int_{w}^{w+1} d v \frac{\theta_{1}^{\prime}(v \mid \tau)}{\theta_{1}(v \mid \tau)}
$$




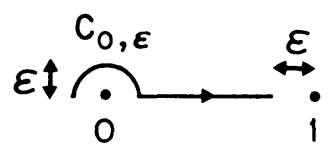

Fig. 4 a

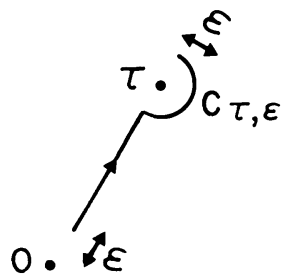

Fig. $4 b$

along the straight path joining $w$ to $w+1$. The path can be deformed to the path depicted in Fig. 4a. Thus

$$
i \varphi_{a}=\lim _{\varepsilon \rightarrow 0^{+}}\left\{\int_{C_{0, \varepsilon}} d v \frac{\theta_{1}^{\prime}(v \mid \tau)}{\theta_{1}(v \mid \tau)}+\int_{\varepsilon}^{1-\varepsilon} d v \frac{\theta_{1}^{\prime}(v \mid \tau)}{\theta_{1}(v \mid \tau)}\right\} .
$$

The second integral vanishes due to the symmetry $\theta_{1}(w \mid \tau)=\theta_{1}(1-w \mid \tau)$, whence the first is given by half of (B.2), with the result $\varphi_{a}=-\pi$. Similarly, for the second equation in (B.1), we introduce the function $\tilde{\theta}_{1}(w \mid \tau)=\exp \left(i \pi w^{2} / \tau\right) \theta_{1}(w \mid \tau)$, antiperiodic as $w \rightarrow w+\tau$. We assume $0<x<1$ and consider the change of phase of $\tilde{\theta}_{1}(w \mid \tau)$ along the straight path from $w$ to $w+\tau$, that we deform as in Fig. $4 \mathrm{~b}$. Since $C_{\tau, \varepsilon}$ has positive orientation we obtain $\varphi_{b}=\pi$. For general $w$, we can deform every straight path from $w$ to $w+1$ (or $w+\tau$ ) to the one in Fig. $4 \mathrm{a}$ (or Fig. 4 b) by making use of (B.2). The result for the phases in (B.1) is then

$$
\begin{aligned}
& \varphi_{a}=\left\{\begin{aligned}
-\pi, & 2 n<y<2 n+1, \\
\pi, & 2 n-1<y<2 n ;
\end{aligned}\right. \\
& \varphi_{b}=\left\{\begin{aligned}
\pi, & 2 m<y<2 m+1, \\
-\pi, & 2 m-1<y<2 m .
\end{aligned}\right.
\end{aligned}
$$

\section{References}

1. Belavin, A. A., Polyakov, A. M., Zamolodchikov, A. B.: Infinite conformal symmetry in twodimensional quantum field theory. Nucl. Phys. B241, 333-380 (1984)

2. Friedan, D., Shenker, S.: The analytic geometry of two-dimensional conformal field theory. Nucl. Phys. B281, 509-545 (1987); The integrable analytic geometry of quantum strings. Phys. Lett. 175B, 287-296 (1986)

3. Eguchi, T., Ooguri, H.: Conformal and current algebra on a general Riemann surface. Nucl. Phys. B282, 308-328 (1987)

4. Cardy, J.: Operator content of two-dimensional conformal invariant theories. Nucl. Phys. B270, 186-204 (1986)

5. Cappelli, A., Itzykson, C., Zuber, J.-B.: The A-D-E classification of two-dimensional conformal invariant theories. Commun. Math. Phys. 113, 1-26 (1987)

6. Dotsenko, Vl.S., Fateev, V.A.: Conformal algebra and multipoint correlation functions in 2d statistical models. Nucl. Phys. B240 [FS12], 312-348 (1984); Four-point correlation functions and operator algebra in $2 \mathrm{~d}$ conformal invariant theories with central charge $\leqq 1$, Nucl. Phys. B251 [FS13], 691-734 (1985); Operator algebra of two-dimensional conformal theories with central charge $C \leqq 1$. Phys. Lett. 154B, 291-295 (1985) 
7. Zamolodchikov, A. B., Fateev, V.A.: Operator algebra and correlation functions in the twodimensional SU $(2) \times \mathrm{SU}(2)$ chiral Wess-Zumino model. Sov. J. Nucl. Phys. 43, 657-664 (1986)

8. Sonoda, H.: Sewing conformal field theories I. Nucl. Phys. B311, 401-416 (1988/89); Sewing conformal field theories II. Nucl. Phys. B311, 417-432 (1988/89)

9. Moore, G., Seiberg, N.: Polynomial equations for rational conformal field theories. Phys. Lett. 212B, 451-460 (1988)

10. Felder, G.: BRST approach to minimal models, ETH preprint (1988); Nucl. Phys. B (to appear)

11. Bagger, J., Nemeschansky, D., Zuber, J.-B.: Minimal model correlation functions on the torus. USC-88/009 (1988)

12. Jayaraman, T., Narain, K.S.: Correlation functions for minimal models on the torus. IC/88/306 (1988)

13. Crnković, Č., Sotkov, G.M., Stanishkov, M.: Minimal models on hyperelliptic surfaces. ISAS-117/EP/88 (1988)

14. Foda, O., Nieunhuis, B.: The Coulomb gas representation of critical RSOS models on the sphere and on the torus. THU-88-34 (1988)

15. Di Francesco, P.: Structure constants for rational conformal field theories. Phys. Lett. 215B, 124-128 (1988)

16. Felder, G., Fröhlich, J., Keller, G.: Braid matrices and structure constants for minimal conformal models, IAS/ETH preprint (1989)

17. Andrews, G.E.: The theory of partitions. Encyclopedia of Mathematics and its Applications. London, Amsterdam, Don Mills (Ontario), Sidney, Tokyo: Addison-Wesley (1976)

18. Bernard, D.: On the Wess-Zumino-Witten model on the torus. Nucl. Phys. B303, 77-93 (1988)

19. Hurwitz, A., Courant, R.: Allgemeine Funktionentheorie und elliptische Funktionen. Berlin: Springer 1929

Communicated by K. Gawedzki

Received January 6, 1989; in revised form February 6, 1989 
